\title{
News from the 2007 San Antonio Breast Cancer Symposium
}

\author{
Janet Fricker
}

1. San Antonio Breast Cancer Symposium (SABCS), San Antonio, TX, December 13-16, 2007

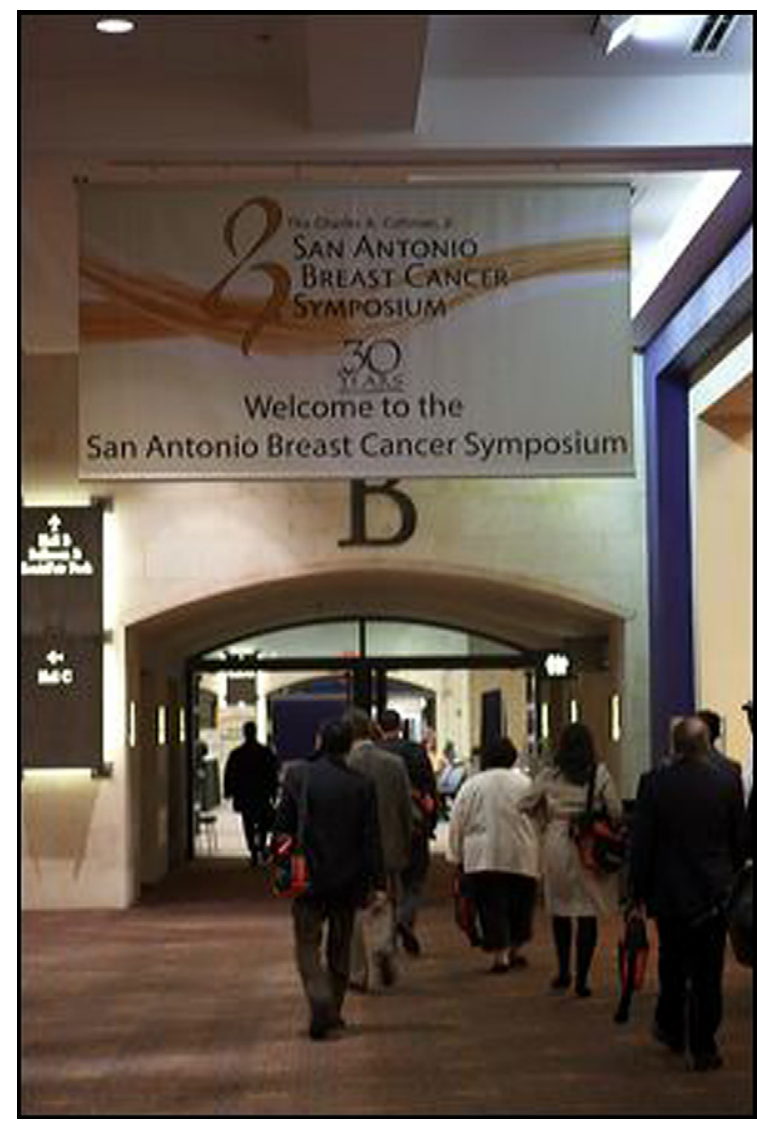

\subsection{Decision to limit tamoxifen to 5 years flawed}

The recommendation to limit adjuvant tamoxifen treatment in breast cancer to 5 years is flawed, concludes the ATLAS study, presented at the 30th San Antonio Breast Cancer Symposium (SABCS) (abstract 48).

The ATLAS trial (Adjuvant Tamoxifen: Longer Against Shorter) is a large international trial comparing the efficacy and safety of 5 years versus 10 years of adjuvant tamoxifen treatment. In the study 11,500 women from 400 international centres who had been taking tamoxifen for 5 years were randomised at year five to continue taking tamoxifen to 10 years or stop treatment.

Presenting the data Sir Richard Peto, from the University of Oxford's Clinical Trials Unit (Oxford, UK), indicated the limitations of the study. Only $59 \%$ of the patients were definitely ER+, the remaining $41 \%$ were untested. Of the untested women around one-quarter are likely to have been ER-, and hence unlikely to experience benefits from tamoxifen. "The upshot is that ATLAS only shows around $72 \%$ of the true effect of tamoxifen," he concluded.

Nevertheless, the study showed that continuing on tamoxifen for an additional 5 years confers about a $12 \%$ reduction in the risk of breast cancer recurrence, compared with stopping treatment. The rate ratio was 0.88 , which was statistically significant with a two-sided $p=0.005$.

For women in the second 5-year period, there's a carry over effect if they were treated with tamoxifen - an additional risk reduction of about $22 \%$ compared with women who had only 5 years of treatment - but this was not statistically significant. All told, there have been 739 recurrences among women allocated to continue tamoxifen and 835 among those stopping treatment.

Although the numbers remain small, said Sir Richard, it is clear that the early adverse results found in the NSABP B 14 study were wrong.

E-mail address: janet.fricker@tesco.net 1574-7891/\$ - see front matter doi:10.1016/j.molonc.2008.01.005 
"The numbers in NSABP B 14 were too small to justify dismissing the important question of whether continuing tamoxifen beyond 5 years could, in the long run, moderately reduce the recurrence rate," said Sir Richard. "The new ATLAS data suggest that longer term use is likely to produce benefits."

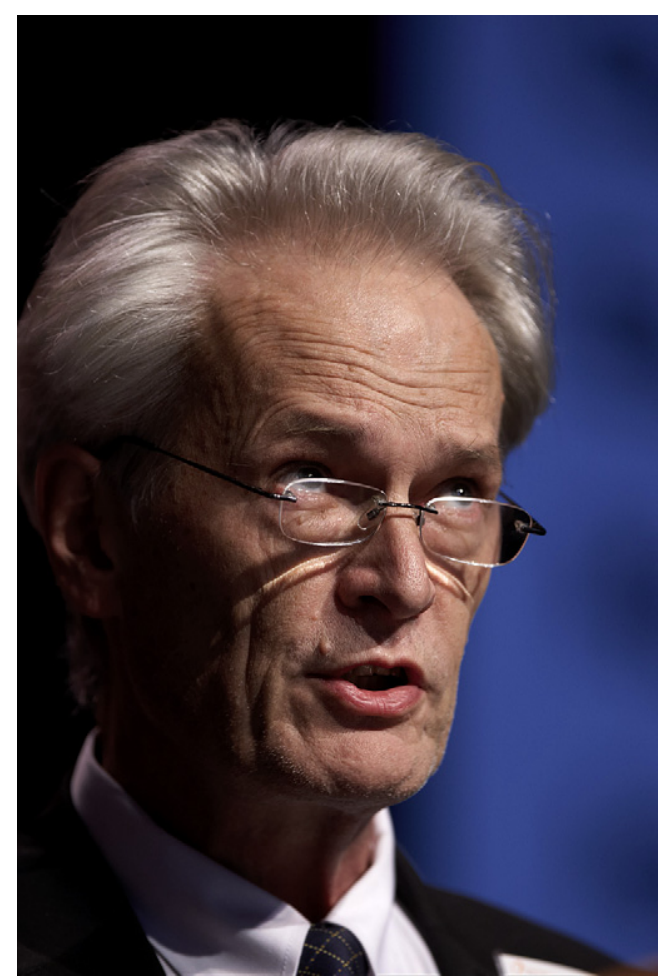

Richard Peto, Courtesy of the San Antonio Breast Cancer Symposium

\subsection{ATAC study: protective effect anastrozole persists beyond treatment}

The 100-month data from the ATAC trial show that even 4 years after stopping adjuvant treatment, the absolute reduction for the risk of breast cancer recurrence continues to increase for anastrozole (Arimidex ${ }^{\circledR}$ ) over tamoxifen, showing for the first time a "carry over" effect for an aromatase inhibitor (abstract 41).

The ATAC (Arimidex, Tamoxifen, Alone or in Combination) 100-month study data, presented at the SABCS and published simultaneously on-line in Lancet Oncology, represent one of the longest and largest breast cancer studies ever undertaken. The study, which started in July 1996, recruited 9366 patients from 381 cancer centres in 21 countries and was designed to establish whether the treatment advantages of anastrozole over tamoxifen, which had already been established with hormone sensitive advanced breast cancer, would be seen in postmenopausal women diagnosed with early stage disease.

In the study women were randomised to receive adjuvant hormonal treatment with anastrozole ( $1 \mathrm{mg}$ daily), tamoxifen (20 mg daily). Analysis at 68 months showed that anastrozole demonstrated greater effect and better tolerability. "But we did not know if the advantages would persist long term," said the study presenter Professor John Forbes from University of Newcastle (Newcastle, New South Wales, Australia).
Results at a median follow-up of 100 months, focusing on the hormone receptor positive subgroup of patients, show that anastrozole reduced the risk of all recurrences by $24 \%$ (HR 0.76 [0.67-0.87]; $p=0.0001)$ and improved the disease free survival by $15 \%$ (HR 0.85 [0.76-0.94]; $p=0.003$ ). An additional result was that anastrozole reduced the risk of distant metastases by $16 \%$ (HR 0.84 [0.72-0.97]; $p=0.022$ ), and reduces the incidence of contra lateral breast cancer by $40 \%$ (OR 0.60 [0.42-0.85]; $p=0.004$ ).

The absolute difference at 5 years was $2.8 \%$ which increased to $4.6 \%$ at 8 years.

"The study shows us that the protective effect of anastrozole is present well beyond completion of treatment, providing an undisputable reason for starting treatment with anastrozole to give women the best chance of staying cancer-free," said Professor Forbes.

One new feature demonstrated by the 100-month data was the discovery that the increased rate of fractures associated anastrozole - a well-known side effect of aromatase inhibitors - vanished once the treatment was stopped.

A difference, however, has yet to be seen in overall survival. "We have to accept that breast cancer is a chronic problem and that if strategies are directed at stopping it coming back survival will look after itself. To put it simply - if a woman doesn't have a breast cancer recurrence she won't die of it," said Forbes.

\subsection{FAT derived stem cells offer new hope in breast reconstruction}

The first clinical trial using stem cells in breast reconstruction for patients who have undergone partial mastectomy showed significantly increased volume of tissue, reports a Japanese study presented at SABCS (abstract 4071).

Although auto-transplantation of adipose tissue has been used for over a century to correct soft-tissue defects, the procedure results in reabsorption of transplanted tissue and unsatisfactory cosmetic results.

The Celution ${ }^{\mathrm{TM}}$ system, developed by Cytori Therapeutics Inc., automates and standardizes the separation and concentration of the patient's own adipose tissue-derived stem and regenerative cells from body fat. The concept behind the technology is that the stem cells produce growth factors and cytokines that enable the growth of blood vessels and successful engraftment of fat.

In the investigator led study, 21 patients, who had previously received breast conservation therapy (19 of whom had also received radiation therapy), underwent 25 stem cell reconstructions. All subjects were deemed to be free of any local recurrence or distant metastasis.

In the procedure fat was harvested from different sites in the body by liposuction. The aspirated tissue was then divided into two equal portions, one of which was used as the primary filler material, while the other was reserved for processing to obtain stem and regenerative cells with the Celution ${ }^{\mathrm{TM}}$ system. The proprietary system works by freeing up the stem and regenerative cells from the connective tissue, separating the cells from the waste material. Finally both portions are combined and injected into the breast void requiring filling.

Using ultra sound, investigators measured the average tissue thickness from the most prominent indentation of the scar to the pectoral muscle. Results show that thickness 
increased from $6 \mathrm{~mm}$ at baseline, to $17 \mathrm{~mm} 1 \mathrm{month}$ after the procedure $(p<0.05)$. Furthermore, at 6 months the thickness was $15 \mathrm{~mm}$, demonstrating that there had not been any significant loss of tissue from the 1-month data. When interviewed $79 \%$ of the patients reported satisfaction with their cosmetic results.

"The procedure helps to lessen the physical and emotional impact of partial mastectomy by permanently restoring the natural appearance of the breast," said the principal investigator Professor Kaoru Kitamura, from Kyushu Central Hospital (Fukuoka, Japan), adding that for one-quarter of women the results were so good that you would not have known they had breast cancer surgery.

During 2008 Cytori plan to support two European trials of the transplant procedure. One will be a multicentre trial involving as many as 70 patients, while the other will be a single-site study involving 20 patients with severe radiation damage and contour defects.

\subsection{Anthracycline drugs: "crazy one-size-fits-all" approach}

Anthracyclines, drugs forming the backbone of adjuvant treatment for breast cancer, only work in a minority of women, reported a SABCS study (abstract 13).

The continued use of the drugs "on a one-size-fits-all approach is just crazy and it's medically dangerous," Dennis Slamon from the University of California, Los Angeles, Jonsson Comprehensive Cancer Center, told on oral session of the meeting.

In the study Slamon and colleagues undertook a systematic review of published data from randomised, controlled adjuvant chemotherapy trials reporting HER2 subtype, and whether or not they contained co-amplification of the topoisomerase II alpha gene (Topo IIa).

Both retrospective and prospective data show that the anthracyclines only benefit women with amplification of both the HER2 receptor and Topo IIa. Among HER2-positive women treated with doxorubicin and cyclophosphamide (Cytoxan), those with a normal or deleted Topo IIa gene had a median survival of 18.2 months. In contrast, women with amplified Topo IIa had a median survival of 38.5 months a difference that was significant at $p=0.004$.

Such dual gene amplifications occur together in only $8 \%$ of breast cancer patients. This means that $92 \%$ of women who receive this toxic drug derive no benefit from it whatsoever. The anthracyclines - such as doxorubicin (Adriamycin) and idarubicin (Idamycin) - are associated with cardiac and bone marrow morbidity and mortality.

"The reality is that there's probably a $25-30 \%$ benefit for a small subgroup while the remaining patients do not benefit," Salmon said.

\subsection{Capecitabine changes treatment landscape in advanced breast cancer}

Adding the oral chemotherapy agent capecitabine (Xeloda) to trastuzumab (Herceptin) and docetaxel increased the median time to disease progression by 5 months in women with

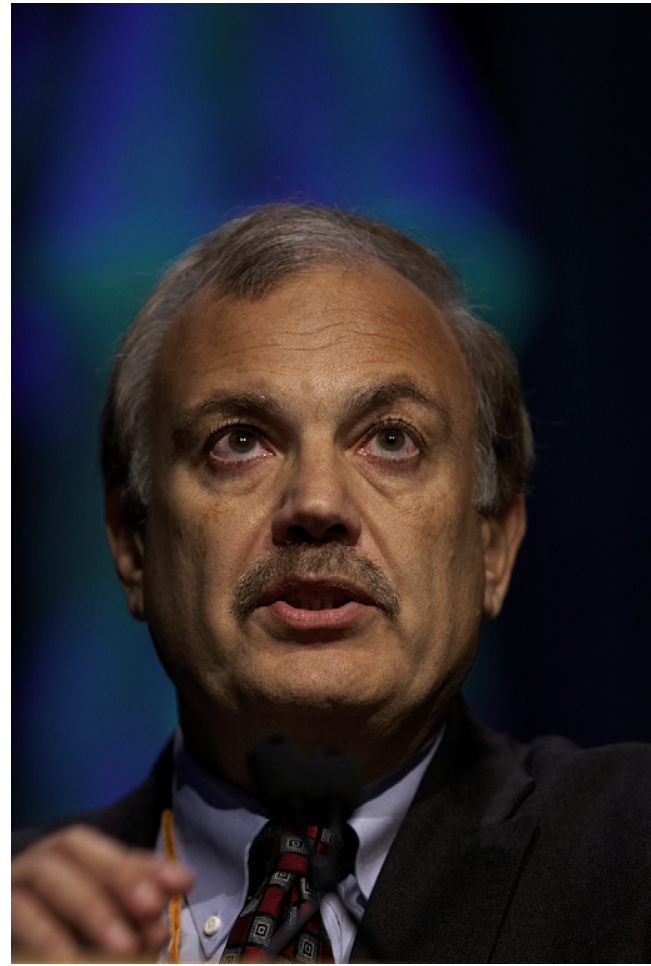

Dennis Slamon, Courtesy of the San Antonio Breast Cancer Symposium

advanced HER2-positive breast cancer, reported a study at SABCS (abstract).

The international, phase 2 CHAT study (Capecitabine, Herceptin and Taxotere) randomised 222 women with HER2-positive locally advanced or metastatic breast cancer to trastuzumab plus docetaxel, with or without capecitabine. Dosing for trastuzumab was with a $8 \mathrm{mg} / \mathrm{kg}$ loading dose, followed by $6 \mathrm{mg} / \mathrm{kg}$ every 3 weeks; docetaxel was given at a dose of $75 \mathrm{mg} / \mathrm{m}^{2}$ in patients receiving the triple drug combination and $100 \mathrm{mg} / \mathrm{m}^{2}$ in the trastuzumab/docetaxel group; capecitabine was administered at a dose of $950 \mathrm{mg} / \mathrm{m}^{2}$ twice daily for the first 14-days of each 3-week cycle.

Results showed that median time to progression (TTP) was $30 \%$ longer, at 18.6 months, in women randomised to capecitabine in addition to trastuzumab and docetaxel compared to those given only trastuzumab plus docetaxel (13.6 months; hazard ratio $0.704 ; 95 \%$ confidence interval 0.51, 0.971; $p=0.0295$ ). Median progression free survival also increased, from 12.8 months with the two-drug combination to 17.9 months when capecitabine was added ( $p=0.0402$ ).

“Trastuzumab's ability to increase survival changed the treatment landscape for patients with advanced breast cancer. Adding capecitabine to the most commonly used first-line regimen of trastuzumab plus a taxane enables patients to live even longer without their disease progressing," said investigator Andrew Wardley, from the Christie Hospital NHS Foundation Trust (Manchester, UK).

He added that as capecitabine is an oral chemotherapy, patients can take it at home so that they did not need to spend additional time in hospital. 